\title{
Optical interferometry image reconstruction contest VIII
}

Mérand, Antoine, Alberdid, Antxon, Kluska, Jacques, Tallon-Bosc, Isabelle, Tallon, Michel, et al.

Antoine Mérand, Antxon Alberdid, Jacques Kluska, Isabelle Tallon-Bosc, Michel Tallon, Éric Thiébaut, Joel Sánchez-Bermudez, Rainer Schödel, Ferréol Soulez, John Young, "Optical interferometry image reconstruction contest VIII," Proc. SPIE 10701, Optical and Infrared Interferometry and Imaging VI, 107011 (9 July 2018); doi: 10.1117/12.2500674 United States 


\title{
Optical Interferometry Image Reconstruction Contest VIII
}

\author{
Antoine Mérand ${ }^{a}$, Antxon Alberdid ${ }^{b}$, Jacques Kluska $^{c}$, Isabelle Tallon-Bosc ${ }^{d}$, Michel Tallon ${ }^{d}$, \\ Éric Thiébaut $^{d}$, Joel Sánchez-Bermudez ${ }^{e}$, Rainer Schödel ${ }^{b}$, Ferréol Soulez ${ }^{d}$ and John Young ${ }^{f}$ \\ ${ }^{a}$ European Southern Observatory, Karl-Schwarzschild-Straße 2, 85748 Garching bei Muenchen, \\ Germany; \\ ${ }^{b}$ IAA-CSIC, Glorieta de la Astronoma, s/n, 18008 Granada, Spain \\ ${ }^{c}$ Instituut voor Sterrenkunde Celestijnenlaan 200D bus 2401 3001, Leuven, Belgium \\ ${ }^{d}$ Univ Lyon, Univ Lyon1, Ens de Lyon, CNRS, Centre de Recherche Astrophysique de Lyon \\ UMR5574, F-69230, Saint-Genis-Laval, France \\ ${ }^{e}$ European Southern Observatory, Alonso de Córdova 3107, Vitacura, Chile \\ ${ }^{f}$ Cavendish Laboratory, Cambridge University, JJ Thomson Ave, Cambridge CB3 0HE, United \\ Kingdom
}

\begin{abstract}
We report on the VIII edition of the imaging reconstruction contest held in the optical/infrared interferometry community. This edition sported a VLTI+CHARA dataset of a simulated image of a star surrounded by a proto-planetary disk hosting a planet. 5 teams responded to the challenge and produced images very close the original image, showing the maturity of image reconstruction techniques for a well sampled $\mathrm{u}, \mathrm{v}$ coverage. The contest was organised by the first author of this article, whereas the following authors provided entries to the contest.
\end{abstract}

Keywords: Optical Interferometry, image reconstruction, contest

\section{INTRODUCTION}

The "Optical Interferometry Image Reconstruction Contest" is organised every two years ${ }^{1-7}$ on the same principles since its first edition in 2004: one or several datasets are produced in the OIFITS format and provided to teams which will attempt to reconstruct images. The contest aims at not only comparing different image reconstruction algorithms, but also to encourage the use of the OIFITS format for optical interferometric data. The contest was announced on March 13th, 2018, on the OLBIN email list*.

\section{DATASET}

This year, only one dataset was provided using the following method. First, OIFITS2 ${ }^{8}$ were generated using the Aspro software. ${ }^{9}$ Since the contest intended to demonstrate the complementarity of the spatial frequency coverage ( $\mathrm{u}, \mathrm{v}$ coverage) of the VLTI and CHARA Array, we chose four different telescope configurations: CHARA $6 \mathrm{~T}$ (that is, using all the available telescopes) as well as the 3 different 4 Auxiliary Telescopes configurations currently offered at VLTI. The declination was chosen to be +9 degrees and realistic observabilities were chosen. Aspro's simulator estimated we could have pointed the object 4 times with CHARA and 8 times with VLTI (on each of the AT configuration) within the observability window (limited by delay line range, object's elevation or domes' shadowing — for VLTI-). Figure 1 shows the resulting coverage.

We did not use a simulated image to generate the data, but rather analytical formulas to generate synthetic visibilities (squared amplitudes and closure phases). The instrument we simulated (MIRC ${ }^{10}$ and PIONIER ${ }^{11}$ ) have low spectral resolution $(\sim 50)$ and we did not introduce chromaticity in the simulated data. Noise was added as follow: each individual $\mathrm{V}^{2}$ or $\mathrm{CP}$ had a gaussian noise, and an overall correlated noise was added for each baseline and each pointing.

Further author information: E-mail: amerand@eso.org

*olbin@univ-grenoble-alpes.fr

Optical and Infrared Interferometry and Imaging VI, edited by Michelle J. Creech-Eakman,

Peter G. Tuthill, Antoine Mérand, Proc. of SPIE Vol. 10701, 107011U · (c) 2018 SPIE

CCC code: $0277-786 \mathrm{X} / 18 / \$ 18 \cdot$ doi: $10.1117 / 12.2500674$ 

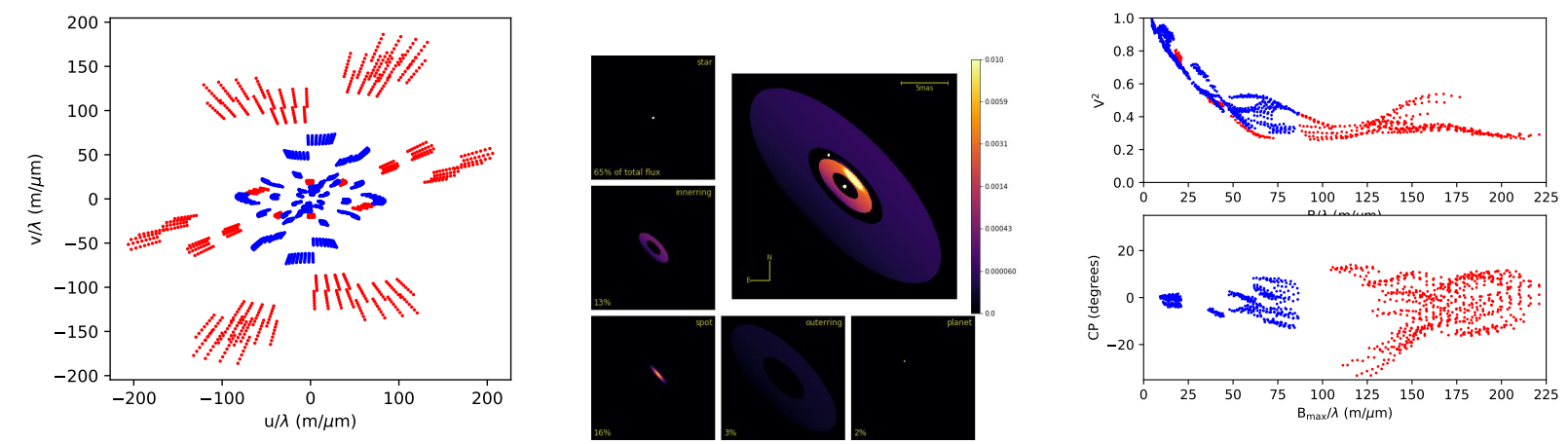

Figure 1. Leftmost panel: $\mathrm{u}, \mathrm{v}$ coverage of the data set. CHARA/MIRC data points are in red, whereas VLTI/PIONIER data points are in blue. Central panels: Model used to generate the synthetic data showing each component described in the text, as well as a synthetic image (large upper right panel) in non linear colour scale. Rightmost panels: $\mathrm{V}^{2}$ (upper) and phase closure (lower) as a function of spatial frequency. Same colour code as the leftmost panel.

The model was composed of 1) a 0.4 mas uniform disk star (65\% of the total flux) 2) an inner ringed disk with a bright rim (29\%) and projection angle of 44.2degrees 3 ) a planet as a $0.3 \mathrm{mas}$ UD at $3.75 \mathrm{mas}$ from the central star and projection angle of 27 degrees $(2 \%)$ and 4$)$ the outer disk extended up to 20mas. See Figure 1 for an overview of the model and the corresponding synthetic image.

The parameters of the model was adjusted to match qualitatively the visibility and closure phases which are observed for young stellar objects in near-infrared interferometry: ${ }^{12}$ the visibilities drop rapidly as the baseline length increase because the disk is rapidly resolved. For longer baselines, the visibility's amplitude reaches a plateau, which height depends on flux ratio between the central (unresolved) star and the disk.

The goal of the contest was set to measure the orientation of the disk and estimate the position of the planet it it was detected, or estimate an upper limit of its flux otherwise.

\section{IMAGE RECONSTRUCTION ENTRIES}

We reproduce in the following sections the text accompanying each submission.

\subsection{Submission from J. Kluska}

We used the MiRA algorithm ${ }^{13}$ together with the SPARCO approach. ${ }^{14}$ It minimises the following cost function: $f=\chi^{2}+\mu f_{\mathrm{rgl}}$, where $f_{\mathrm{rgl}}$ is the cost function of the regularisation and $\mu$ is the regularisation weight. The SPARCO approach separates a geometrical model of one component of the observed object to reconstruct the image of the rest. We used the quadratic smoothness regularisation as it is retrieving the images well ${ }^{15}$ and helps to not fall into local minima. We divided our approach into 3 steps. At step 1 we selected the field-of-view of the image to be roughly 3 times larger than the reconstructed morphology and the pixel-size to be significantly smaller that the highest angular resolution reached by the longest baseline. The pixel size was therefore chosen to be 0.1 mas and the image is made of $256 \times 256$ pixels. For each step we took a different fixed model and the image reconstruction was therefore made on different component of the object. We tuned the regularisation weight at each step in order to retrieve an image of a good quality $\left(10^{8}, 10^{9}\right.$ and $10^{4}$ for each step respectively).

Here we describe the 3 steps:

1. model = point source. Given the dataset and the instructions we assumed that there is a non-resolved star in the object. We modelled it as a point source and performed an image reconstruction of its environment. We fitted the flux of the point source bu performing a grid with different flux ratios and choosing the one that has the smallest value of the global cost function $(f)$ consisted in assuming that there is an unresolved star, in order to reconstruct the environment. The recovered image presented an elongated 
emission with a bright spot at coordinates $\mathrm{x} \sim 1.8$ mas and $\mathrm{y} \sim 3.3$ mas. There was also some flux present very close to the star itself as it was marginally resolved.

2. model $=$ uniform disk + point source. In the second step we modelled the the central star as a uniform disk of a diameter of 0.45 mas (to account for the flux surrounding the star at step 1) and a point source for the planet at the coordinates mentioned above. We manually tuned the flux of the star and the planet to fit the data. This step allowed the regularisation to act on the image of the disk only as the presence of the star and the planet would break the effect of the regularisation (as they have a high surface brightness and a sharp morphology compared to the extended disk flux).It allowed an image of the disk of better quality.

3. model = image of the disk. In the last step we used the image of the disk obtained at step 2 to reconstruct the two point sources and relax the constraints we put by modelling them with geometrical models. The flux ratio was the complementary one to the fluxes of the star and planet at step 2 .

The final image is the addition of the image of step 2 with the image of step 3 with the correct flux ratio.

The position of the planet was measured at step 1, with the error bars being the extension of the flux at this position $(\Delta \mathrm{x}=1.8 \pm 0.3$ mas; $\Delta \mathrm{y}=3.3 \pm 0.3 \mathrm{mas})$. The position angle of the disk was measured at step 2 by fitting a 2D-Gaussian the amplitude of the Fourier transform of the image of the disk. The result is $45.5^{\circ} \pm 2^{\circ}$.

\subsection{Submission from É. Thiébaut}

Reconstruction were carried out with $\mathrm{MiRA}^{13 \dagger}$, using the power spectrum (OI-VIS2) and phase closure data (OI-T3PHI) of the provided dataset. The image reconstruction settings were as follows: pixel size is $\delta \theta=0.1$ mas, synthesized field of view $\Omega=20$ mas (hence restored images are $200 \times 200$ pixels). The maximum sampled frequency is about 200 Mega cycles which corresponds to an angular resolution of 0.5mas; hence images have a fair amount of super-resolution. Positivity and normalisation constraints were applied (such that the sum of pixel values is equal to 1). The different reconstructions were all started with an initial image given by an unresolved source centered in the field of view. An hyperbolic edge preserving smoothness ${ }^{16}$ was used as regularisation term with transition threshold parameter $\tau$ and relative weight $\mu$. Whatever the combination of the regularisation parameters, the restored images (see Fig. 1) clearly show a bright central star surrounded by a disk (tilted with respect to the observer, so only a fraction of the inner edge is seen) and a faint unresolved point-like source (North-East from the star) believed to be an exo-planet. There are a few other point-like structures inside the void surrounding the star but much fainter (the brightest one is 3 times fainter than the supposed exo-planet). The planet is at position angle PA $=28.1 \pm 0.2$ (from North toward East), and separation $\rho=3.71 \pm 0.01$ mas. The integrated flux of the planet is $2.0 \pm 0.1 \%$ of the total flux while the integrated flux of the star is $65.6 \pm 0.2 \%$ of the total flux. Most of the remaining flux is in the disk-like structure. These parameters does not much depend on the regularization settings. The major axis of the proto-planetary disk has a position angle of about $\mathrm{PA}_{\text {maj }}$ $=45 \pm 1$ and a length of $6.5 \pm 0.5$ mas. Regularization parameters $\mu=3^{4}$ and $\tau=5 \times 10^{4}$ yield one of the best image and was chosen for the challenge.

\subsection{Submission from J. Sanchez-Bermudez, A. Alberdi and R. Schödel}

In order to recover the morphology from the delivered MIRC and PIONIER observables, we used two of the software available to the community: BSMEM and SQUEEZE. Our reconstruction process first consisted in combine all the data sets into a single OIFITS file. In order to have the same wavelength information for both MIRC and PIONIER, we performed a wavelength interpolation in the MIRC data set at PIONIER wavelengths. Since the source is considered a grey body (i.e., the morphology is the same at all the sampled wavelengths) we did not performed a chromatic reconstruction of the delivered data set. After the combination, we used SQUEEZE to perform the first reconstruction. This is a Monte Carlo - Markov Chain software that allows us to compute parallel reconstruction chains with a large variety of regularisers. For this exercise, we computed 10 different chains (with 250 iterations each one) to find the best image that suits the interferometric observables. We used a Laplacian regulariser with an hyper parameter value of $?=100$ (other combinations of regularisers and

\footnotetext{
${ }^{\dagger}$ MiRA version 2.0.0b available at https://github.com/emmt/MiRA
} 
hyper parameters were tested, but no significant morphological changes were observed in the images); the pixel scale was set to $0.1 \mathrm{mas} /$ pixel (since the maximum angular resolution of the array was of $\theta=0.5$ mas) over a pixel grid of 40 mas. SQUEEZE converged for all the ten different chains. The morphology of the object shows a circum-stellar disk/rim around a central star and a secondary source close to the north-east edge of the rim.

Additionally to these three morphological components, we observed several other point-like sources in the field-of-view. Since one of the objectives of the contest is to find the planetary component in the image, we had to refine our reconstruction to discard spurious components associated to systematics, noise, or to the secondary lobes of the synthesised beam. To refine the SQUEEZE solution, for the ten different images, we filter the three principal morphological components by masking all the other emission/noise in the field-of-view. With these masked maps, we then used BSMEM for a second iteration to find the best image. BSMEM uses a gradientdescent method to perform the minimisation process for the reconstruction. For this software, the regularisation uses mainly Gull-Skilling entropy and the hyper parameter value is selected automatically for the user. We performed 60 iterations for the reconstruction. With this setup, we managed to find images that fit, with highdegree of accuracy, the squared visibilities and closure phases. Finally a raw-mean image was created with the ten reconstructed frames. An additional convolved image with a point-spread-function of 0.5 mas (FWHM) was also created. Figure 1 displays the best-fit reconstructed images (raw and convolved), with the morphological elements labeled; Figure 2 shows the interferometric observables reconstructed from the raw image, together with the squared differences that result from the comparison with the data. Our results support the following conclusions over the reconstructed images:

- Three main components are detected: (a) a central source with $\sim 65.8 \%$ of the total flux in the image; (b) a circumstellar rim with $\sim 29.4 \%$ of the flux and a secondary companion, plausible a planet, with $\sim 2.2 \%$ of the flux. The rest of the flux in the image was spread over the field-of-view with noise peaks of the order of $\sim 5 \times 10^{-4} \%$ of the total flux ( $\sim 10^{3}$ times fainter than the image peak).

- The circum stellar rim is elongated with the projected position angle (PA) of the semi-major axis at $\sim 47$ degrees (East of North). The rim also exhibits a non-uniform brightness distribution with the maximum located at PA 309 degrees. The brightness distribution of the rim decreases quickly from the peak to the rest of the rim profile. Uncertainties in the observables, the sparse $\mathrm{u}, \mathrm{v}$ coverage, and the limitations in the reconstruction make clumpy the emission along the rim profile. At a $\mathrm{PA}=139$ degrees no emission is detected from the rim.

- The plausible planetary component is detected nearby the outer edge of the rim at a separation of $\sim 3.8$ mas form the central source and at a PA 28 degrees. During the first reconstruction, two additional point-like sources were found, one inside the rim cavity and a second one in the depleted emission area of the rim. At first sight, we thought that one of them could be the sought planetary companion. Nevertheless, after revising the secondary lobes of the synthesised beam, we noticed that these additional point-like sources lie at the loci of two of the secondary lobes. Therefore, we discarded them as real sources. This hypothesis was confirmed after the second reconstruction, when only a remanent of them appeared in the images.

- The peak of the squared differences between the data and the interferometric observables from the meanreconstructed images are $5 \times 10^{-5}$ for the $\mathrm{V}^{2}$ and $0.1 \mathrm{deg}$ for the closure phases of both, the MIRC and PIONIER data sets.

\section{4 submission from J. Young}

The BSMEM (BiSpectrum Maximum Entropy Method) software was first written in 1992 to demonstrate image reconstruction from optical aperture synthesis data. It has been extensively enhanced and tested since then. BSMEM was adapted during 2015-2016 to work with the "OImaging" graphical user interface developed at JMMC. The 2018 contest entry was produced with v2.1.1 of BSMEM ${ }^{\ddagger}$

\footnotetext{
${ }^{\ddagger}$ BSMEM contains proprietary software, but may be downloaded for non-commercial use subject to a Creative Commons Attribution-NonCommercial-NoDerivatives 4.0 International License - see http://www.mrao.cam.ac.uk/research/optical-interferometry/bsmem-software/
} 
The BSMEM algorithm applies a fully Bayesian approach to the inverse problem of finding the most probable image given the evidence, making use of the Maximum Entropy approach to maximize the posterior probability of an image. The algorithm uses a trust region method with non-linear conjugate gradient steps to minimise the sum of the $\log$ (likelihood $\chi^{2}$ of the data given the image and a regularization term expressed as the Gull-Skilling entropy $\sum_{k} I_{k}-M_{k}-I_{k} \log \left(I_{k} / M_{k}\right)$. The model image $M_{k}$ is usually chosen to be a Gaussian, a uniform disk, or a delta-function centered in the field of view, which conveniently fixes the location of the reconstructed object (the bispectra and powerspectra being invariant to translation). This type of starting model also acts as a support constraint by penalising the presence of flux far from the centre of the image.

The oifitslib utility programs were used to merge the CHARA and VLTI contest datasets into a single OIFITS file, and to remove the complex visibility and triple product amplitude data. An initial reconstruction was made from the combined dataset using a simple circular Gaussian prior model. The approximate orientation and axial ratio of the protoplanetary disk were inferred from the initial reconstruction, and a new model image consistent with these parameters was created. The model image was created in LITpro and comprised a compact circular Gaussian, representing the star, plus a flattened disk encompassing all of the believable circumstellar disk flux in the initial reconstruction (the model image can be found in the second HDU of the submitted FITS file). The submitted reconstruction was made using this second prior image.

Given the systematic errors deliberately introduced into the contest dataset, BSMEM's automatic hyperparameter estimation was not used. The optimum regularization weight was chosen using the "L-curve" approach. The final reduced chi-squared of the submitted image was 1.23.

From the submitted image, the position angle ( $\mathrm{N}$ through $\mathrm{E}$ ) of the protoplanetary disk is 43 degrees. The supposed planet is 3.76 mas from the central star, at position angle ( $\mathrm{N}$ through $\mathrm{E}$ ) 28.6 degrees.

\section{5 submission from M. Tallon, I. Tallon-Bosc, É. Thiébaut and F. Soulez}

We attempted to tackle the imaging contest 2018 by using model fitting with LITpro ${ }^{17}$ and to get a normalised image from the model determined in the Fourier plane from fitting the provided OI-VIS2 and OI-T3PHI data. The best solution was built from these elements:

- a central star gathering $65.3 \pm 2.5 \%$ of the total flux;

- a component (planet) at separation $3.73 \pm 0.01$ mas and position angle $\mathrm{PA}=27.3 \pm 0.3$ degrees (i.e. NorthEast quadrant) gathering $1.9 \pm 0.1 \%$ of the total flux;

- an elongated proto-planetary disk, with a major axis at position angle $\mathrm{PA}=42.4 \pm 0.3$ degrees, the length of the elongation found to be $6.05 \pm 0.05$ mas;

- an elongated gaussian halo, with a major axis at position angle $\mathrm{PA}=47 \pm 2$ degrees and a FWHM of $15.4 \pm 0.6$ mas along this direction, gathering $4.2 \pm 0.25 \%$ of the total flux.

The difficulty was to calculate the image from the model while controlling the artefacts brought by the FFT. The model is indeed perfectly determined in the Fourier plane whereas the parameters for the image calculation, like the pixel size or the resolution, are arbitrary: this is a major difference between model fitting and image reconstruction. The pixel size of the image has been chosen equal to 0.1 mas, small enough to prevent any pixelization effect. The image has then been convolved by a gaussian with a FWHM of 0.16 mas to reduce the truncation effect in the Fourier plane. That gives a resolution of about 0.3 mas, three times better than the diffraction limit of the measurements.

From this process, we can draw out some interesting remarks:

- Fitting the star and the planet by gaussians gives the same FWHM for both: 0.250 .05 mas. This suggests that the data were computed from an image obtained by convolving a perfect object with a gaussian of this width. It is somehow confusing since it looks like we modelize the making of the image instead of the shape of the sources in the sky. 


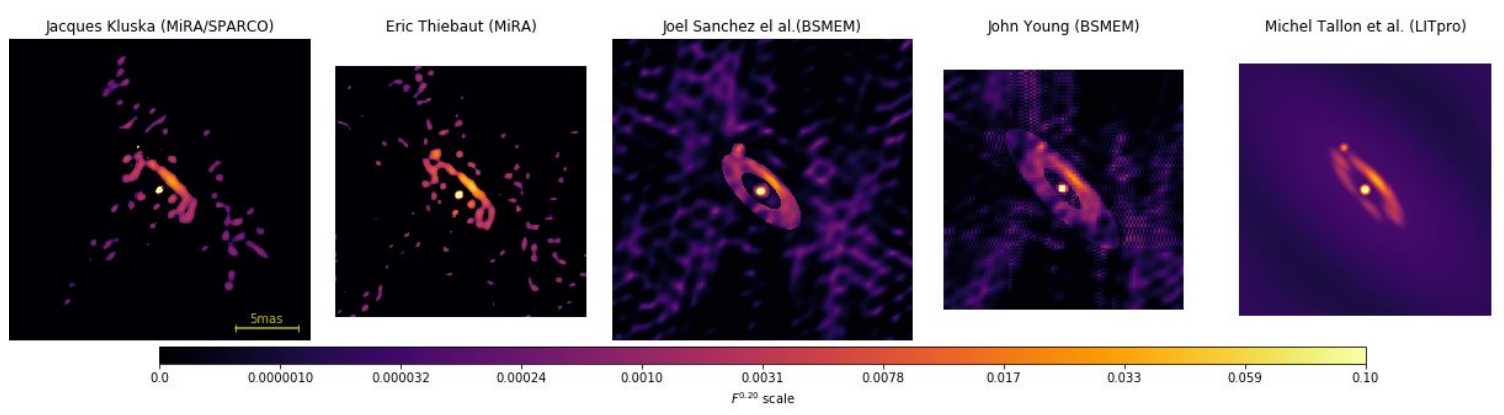

Figure 2. All reconstructed images at the same spatial and color scales. The color scale is a power law in order to show the lowest surface brightness details.

- For modelling the proto-planetary disk, we have developed a new model: the intensity along a circle is represented by a set of points interpolated by cubic splines. This circle is then stretched, oriented and convolved by a kernel (here a gaussian). This yields a very flexible adjustment.

- The planet is obvious in the data. For example, if the planet is removed from the model, the fitter forces the disk to move and to produce a spot exactly at the location of the planet, by activating one cubic spline of the disk.

\section{RESULTS AND CONCLUSIONS}

All reconstructed images are shown on Figure 2. From the previous descriptions of the entries, it is clear that all resulting in a correct measurement of the disk orientation and planet position. We came up with two additional criteria:

- Was the gap between the inner and outer disks detected, at the correct position? Yes for Kluska, Thiébaut and Sanchez-Bermudez et al.

- Was the inner disk reconstructed in its entirety? Yes for Sanchez-Bermudez et al., Young and Tallon et al.

Considering that only one entry satisfies the two criteria (Sanchez-Bermudez et al.), this entry was deemed the winner of Optical Interferometry Image Reconstruction Contest VIII.

In Conclusions, all images managed well to reproduce the original model used for the data synthesis. We can also note that some images were produced using novel techniques. For example, the use of one algorithm to produce a first image which is then used to feed a second algorithm (J. Sanchez-Bermudez et al.). Additionally, we can see the advent of approaches not limited to "pixel-based" modeling of the image: J. Kluska used some analytical components, while M. Tallon et al. used a fully analytical modeling of the visibility. Interestingly, only the analytical modeling, using LITpro, was able to model the low surface brightness, extended outer disk.

\section{REFERENCES}

[1] Lawson, P. R., Cotton, W. D., Hummel, C. A., Monnier, J. D., Zhao, M., Young, J. S., Thorsteinsson, H., Meimon, S. C., Mugnier, L. M., Le Besnerais, G., Thiebaut, E. M., and Tuthill, P. G., "An interferometry imaging beauty contest," in [New Frontiers in Stellar Interferometry], Traub, W. A., ed., Proceedings of the SPIE 5491, 886 (Oct. 2004).

[2] Lawson, P. R., Cotton, W. D., Hummel, C. A., Baron, F., Young, J. S., Kraus, S., Hofmann, K.-H., Weigelt, G. P., Ireland, M., Monnier, J. D., Thiébaut, E., Rengaswamy, S., and Chesneau, O., "2006 interferometry imaging beauty contest," in [Society of Photo-Optical Instrumentation Engineers (SPIE) Conference Series], Proceedings of the SPIE 6268, 62681U (June 2006). 
[3] Cotton, W., Monnier, J., Baron, F., Hofmann, K.-H., Kraus, S., Weigelt, G., Rengaswamy, S., Thiébaut, E., Lawson, P., Jaffe, W., Hummel, C., Pauls, T., Schmitt, H., Tuthill, P., and Young, J., "2008 imaging beauty contest," in [Optical and Infrared Interferometry], Proceedings of the SPIE 7013, 70131N (July 2008).

[4] Malbet, F., Cotton, W., Duvert, G., Lawson, P., Chiavassa, A., Young, J., Baron, F., Buscher, D., Rengaswamy, S., Kloppenborg, B., Vannier, M., and Mugnier, L., "The 2010 interferometric imaging beauty contest," in [Optical and Infrared Interferometry II], Proceedings of the SPIE 7734, 77342N (July 2010).

[5] Baron, F., Cotton, W. D., Lawson, P. R., Ridgway, S. T., Aarnio, A., Monnier, J. D., Hofmann, K.-H., Schertl, D., Weigelt, G., Thiébaut, E., Soulez, F., Mary, D., Millour, F., Vannier, M., Young, J., Elias, N. M., Schmitt, H. R., and Rengaswamy, S., "The 2012 interferometric imaging beauty contest," in [Optical and Infrared Interferometry III], Proceedings of the SPIE 8445, 84451E (July 2012).

[6] Monnier, J. D., Berger, J.-P., Le Bouquin, J.-B., Tuthill, P. G., Wittkowski, M., Grellmann, R., Müller, A., Renganswany, S., Hummel, C., Hofmann, K.-H., Schertl, D., Weigelt, G., Young, J., Buscher, D., Sanchez-Bermudez, J., Alberdi, A., Schoedel, R., Köhler, R., Soulez, F., Thiébaut, É., Kluska, J., Malbet, F., Duvert, G., Kraus, S., Kloppenborg, B. K., Baron, F., de Wit, W.-J., Rivinius, T., and Merand, A., "The 2014 interferometric imaging beauty contest," in [Optical and Infrared Interferometry IV], Proceedings of the SPIE 9146, 91461Q (July 2014).

[7] Sanchez-Bermudez, J., Thiébaut, E., Hofmann, K.-H., Heininger, M., Schertl, D., Weigelt, G., Millour, F., Schutz, A., Ferrari, A., Vannier, M., Mary, D., and Young, J., "The 2016 interferometric imaging beauty contest," in [Optical and Infrared Interferometry and Imaging V], Proceedings of the SPIE 9907, 99071D (Aug. 2016).

[8] Duvert, G., Young, J., and Hummel, C. A., "OIFITS 2: the 2nd version of the data exchange standard for optical interferometry," A\&̈A 597, A8 (Jan. 2017).

[9] Bourgès, L., Mella, G., Lafrasse, S., and Duvert, G., "ASPRO 2: Astronomical Software to PRepare Observations." Astrophysics Source Code Library (Oct. 2013).

[10] Monnier, J. D., Berger, J.-P., Millan-Gabet, R., and ten Brummelaar, T. A., "The Michigan Infrared Combiner (MIRC): IR imaging with the CHARA Array," in [New Frontiers in Stellar Interferometry], Traub, W. A., ed., Proceedings of the SPIE 5491, 1370 (Oct. 2004).

[11] Berger, J.-P., Zins, G., Lazareff, B., Lebouquin, J.-B., Jocou, L., Kern, P., Millan-Gabet, R., Traub, W., Haguenauer, P., Absil, O., Augereau, J.-C., Benisty, M., Blind, N., Bonfils, X., Delboulbe, A., Feautrier, P., Germain, M., Gillier, D., Gitton, P., Kiekebusch, M., Knudstrup, J., Lizon, J.-L., Magnard, Y., Malbet, F., Maurel, D., Menard, F., Micallef, M., Michaud, L., Morel, S., Moulin, T., Popovic, D., Perraut, K., Rabou, P., Rochat, S., Roussel, F., Roux, A., Stadler, E., and Tatulli, E., "PIONIER: a visitor instrument for VLTI," in [Optical and Infrared Interferometry II], Proceedings of the SPIE 7734, 773435 (July 2010).

[12] Lazareff, B., Berger, J.-P., Kluska, J., Le Bouquin, J.-B., Benisty, M., Malbet, F., Koen, C., Pinte, C., Thi, W.-F., Absil, O., Baron, F., Delboulbé, A., Duvert, G., Isella, A., Jocou, L., Juhasz, A., Kraus, S., Lachaume, R., Ménard, F., Millan-Gabet, R., Monnier, J. D., Moulin, T., Perraut, K., Rochat, S., Soulez, F., Tallon, M., Thiébaut, E., Traub, W., and Zins, G., "Structure of Herbig AeBe disks at the milliarcsecond scale . A statistical survey in the H band using PIONIER-VLTI," A 8 A 599, A85 (Mar. 2017).

[13] Thiébaut, E., "MIRA: an effective imaging algorithm for optical interferometry," in [Optical and Infrared Interferometry], Proceedings of the SPIE 7013, 70131I (July 2008).

[14] Kluska, J., Malbet, F., Berger, J.-P., Baron, F., Lazareff, B., Le Bouquin, J.-B., Monnier, J. D., Soulez, F., and Thiébaut, E., "SPARCO : a semi-parametric approach for image reconstruction of chromatic objects. Application to young stellar objects," A\&A 564, A80 (Apr. 2014).

[15] Renard, S., Thiébaut, E., and Malbet, F., "Image reconstruction in optical interferometry: benchmarking the regularization," A\&A 533, A64 (Sept. 2011).

[16] Thiébaut, É. and Young, J., "Principles of image reconstruction in optical interferometry: tutorial," Journal of the Optical Society of America A 34, 904 (June 2017).

[17] Tallon-Bosc, I., Tallon, M., Thiébaut, E., Béchet, C., Mella, G., Lafrasse, S., Chesneau, O., Domiciano de Souza, A., Duvert, G., Mourard, D., Petrov, R., and Vannier, M., "LITpro: a model fitting software for optical interferometry," in [Optical and Infrared Interferometry], Proceedings of the SPIE 7013, 70131J (July 2008). 